\title{
Doppler velocimetry of normal human fetal venous intrapulmonary branches
}

\author{
J. A. M. Laudy, N. T. C. Ursem, P. G. H. Mulder* and J. W. Wladimiroff \\ Department of Obstetrics and Gynecology, University Hospital Rotterdam; "Institute of Epidemiology and \\ Biostatistics, Erasmus University Medical School, Rotterdam, The Netherlands
}

Key words: COLOR DOPPLER, DOPPLER VELOCIMETRY, HUMAN FETUS, PULMONARY VENOUS BLOOD FLOW, REPEATABILITY

\begin{abstract}
Objectives To describe the nature of flow velocity waveforms from fetal middle and distal venous pulmonary branches in the second half of normal pregnancy in relation to gestation, and to test repeatability and interrelationships of flow velocity waveform recordings from proximal, middle and distal venous pulmonary branches.
\end{abstract}

Design Cross-sectional study.

Subjects/methods A total of 111 normal singleton pregnancies between 20 and 40 weeks' gestation were studied using a color-coded Doppler ultrasound system. Pulmonary waveforms were obtained at the level of the fetal cardiac four-chamber view. Repeatability was tested from two recordings at 15 -min time intervals in 25 separate normal pregnancies.

Results The nature of middle and distal venous pulmonary flow velocity waveforms was comparable with that of proximal waveforms. Acceptable repeatability of pulmonary venous flow velocity waveforms with coefficients of variation below $15 \%$ was established for nearly all velocity parameters and their ratios. A gestational age-dependent change was found for all flow velocity waveform parameters including pulsatility index for veins at both middle and distal venous levels. Significant inter-pulmonary changes were observed for nearly all pulmonary venous waveform parameters.

Conclusions It is speculated that increase in volume flow and venous pulmonary pressure gradient plays a role in gestational age-dependent changes, whereas changes in vessel diameter and distance between the heart and more distal venous pulmonary vessels are responsible for interpulmonary changes.

\section{INTRODUCTION}

Our knowledge of the fetal pulmonary circulation is primarily derived from invasive animal studies, particularly in lambs ${ }^{1-3}$. The venous pulmonary blood flow in particular has mainly been studied postnatally both in humans and animals ${ }^{4-6}$. Although hemodynamic indices from all these different studies may subsequently be extrapolated to the human fetus, caution should be applied, since the distribution of the fetal circulation and subsequent fetal flow patterns may be different ${ }^{7}$.

The introduction of color-coded Doppler systems has led to identification of the human fetal venous pulmonary circulation $^{8-10}$. Consequently, non-invasive Doppler studies of the human fetal venous pulmonary circulation with emphasis on the pulmonary venous drainage into the left atrium have been reported ${ }^{11-13}$. However, to our knowledge, information on more distal human fetal venous pulmonary hemodynamics is not available. Doppler velocimetry of proximal, middle and distal parts of the venous pulmonary circulation may improve our knowledge of the total human fetal pulmonary vascular system under normal and abnormal circumstances, such as in pulmonary hypoplasia. We therefore investigated, during the second half of normal singleton pregnancy:

(1) The repeatability of flow velocity waveforms from proximal, middle and distal venous pulmonary branches;

(2) The nature and gestational age-dependency of middle and distal venous pulmonary flow velocity waveforms (data on the nature and gestational age-dependency of proximal venous pulmonary flow velocity waveforms have been reported earlier ${ }^{11}$ );

(3) The relationships between flow velocity waveforms from proximal, middle and distal venous pulmonary branches.

Correspondence: Professor J. W. Wladimiroff, Department of Obstetrics and Gynecology, University Hospital Rotterdam, Erasmus University, School of Medicine and Health Sciences, Dr Molewaterplein 40, 3015 GD Rotterdam, The Netherlands 


\section{PATIENTS AND METHODS}

\section{Study subjects}

A total of 111 non-smoking women with a normal singleton pregnancy consented to participate in this crosssectional study. Women were recruited from the routine antenatal clinic at the University Hospital Rotterdam. The study protocol was approved by the Hospital Ethics Committee. Gestational age at entering the study ranged between 20 and 40 weeks (median 30 weeks) and maternal age varied between 18 and 46 years (median 28 years). Pregnancy duration was determined from the last reliable menstrual period and confirmed or adjusted by ultrasonic measurement of the fetal crown-rump length (8-12 weeks) or fetal biparietal diameter (12-20 weeks). None of these pregnancies was associated with congenital anomalies, including lung pathology. All pregnancies were uneventful, and had normal fetal biometry and normal amniotic fluid volumes. As expected, $80 \%$ of the fetal birth weights were situated between the 10th and 90th centiles according to the Kloosterman tables, adjusted for maternal parity and fetal $\operatorname{sex}^{14}$. Women were selected according to ten gestational age subgroups (20-22, . ., 38-40 weeks), to guarantee a homogeneous distribution. Each woman was included in the study only once.

\section{Recording technique}

Ultrasound Doppler studies were performed using a Toshiba SSH 140 machine with combined transabdominal real-time, color and pulsed Doppler imaging facilities (Toshiba Corp., Medical Systems Division, Tokyo, Japan). The carrier frequency was $5 \mathrm{MHz}$ (real-time) and $3.75 \mathrm{MHz}$ (Doppler). The spatial peak temporal average power output was less than $100 \mathrm{~mW} / \mathrm{cm}^{2}$ in both imaging and Doppler modes, according to the manufacturer's specifications.

After obtaining a clear sonographic transverse crosssection of the fetal chest at the level of the cardiac fourchamber view, color Doppler was used to visualize the pulmonary circulation. Depending on fetal position, the right or left lung was examined. First, the pulsed Doppler sample volume $(0.1-0.3 \mathrm{~cm})$ was placed over the most prominent vein, just proximal to the entrance into the left atrium $^{11}$. The sample volume was then moved to a point in the middle of the fetal lung, at equal distance from the outer border of the fetal heart and the inner thoracic wall. Venous pulmonary blood flow velocity waveforms were collected at this point and from the most distal branch of the pulmonary vein, as close as possible to the fetal inner thoracic wall.

The angle between the Doppler cursor and the assumed blood flow direction was always kept below $20^{\circ}$. The highpass filter was set at $70-100 \mathrm{~Hz}$. Recordings were performed with the woman in the semirecumbent position and during fetal apnea, since the latter may modulate the flow velocity waveform ${ }^{15}$. All flow velocity waveforms were recorded on hard copies. Waveform analysis was performed by one examiner (J.A.M.L.) using a microcomputer
(Commodore $386 \mathrm{CX}$ ) linked to a graphics tablet. At least three consecutive flow velocity waveforms with the highest velocity and of similar appearance were used to calculate the different parameters in each vessel. In each woman, the following waveform parameters from the proximal, middle and distal branch of the pulmonary vein were determined: time-averaged maximum velocity $(\mathrm{TAV} ; \mathrm{cm} / \mathrm{s})$, peak systolic velocity $(\mathrm{cm} / \mathrm{s})$, peak diastolic velocity $(\mathrm{cm} / \mathrm{s})$, end-diastolic velocity $(\mathrm{cm} / \mathrm{s})$, peak systolic/peak diastolic ratio (S/D ratio) and pulsatility index for veins (PIV = (peak systolic velocity - end-diastolic velocity)/TAV) according to Gosling and $\mathrm{King}^{16}$.

Fetal heart rate (FHR; beats/min) was calculated from the time interval (ms) between the onset of the systolic velocity component of two successive venous pulmonary flow velocity waveforms.

\section{Statistical analysis}

Reference centiles (P5, P50 and P95) were constructed ${ }^{17}$ and, if necessary, the standard deviation (SD) was modelled as a function of gestational age ${ }^{18}$. To find the 50th centile (P50), linear regression was used to establish how the outcome variable was related to gestational age. If there was no significant relationship, the mean of the outcome variable $y$ was used. Otherwise, a straight line or quadratic equation was used. If the distribution of $y$ depending on gestational age was skewed, a logarithmic transformation was applied and this transformed variable was modelled again. Further, it was checked whether the absolute residuals depended on gestational age. If so, straight line regression was used to model the absolute value of the residuals and, from this model, a regression equation for the SD, depending on gestational age, was derived. Using this residual SD, the 5th (P5) and 95th (P95) centiles were constructed. In cases of a quadratic relationship, the age range with significant increase or decrease of the outcome variable was found, using the first derivative. We also tested whether the relationship between the outcome variable $y$ and gestational age was influenced by FHR, by adding FHR into the regression model.

The paired $t$ test was applied to establish the difference in mean values of all velocity parameters between proximal and middle, proximal and distal, and middle and distal venous pulmonary branches. We also tested whether these mean differences were related to gestational age. The level of statistical significance was set at $p<0.05$.

\section{Repeatability}

To examine the repeatability of venous pulmonary branch flow velocity waveform recordings, Doppler waveforms from proximal, middle and distal venous pulmonary branches were collected in 25 separate women with an uncomplicated singleton pregnancy. Pregnancy duration ranged between 22 and 26 weeks in 15 cases and between 32 and 36 weeks in ten cases. In each of the 25 fetuses, two recordings were made from each of the Doppler sampling 
locations at a time interval of approximately $15 \mathrm{~min}$. Each recording resulted in at least three waveforms per hard copy. These hard copies did not reveal the identity or gestational age of the patient, nor the date or time of recording. They were coded by number, shuffled into random order and analyzed. Both Doppler recording and waveform analysis were performed by the same investigator (J.A.M.L.).

For assessment of repeatability, the mean differences and SD of differences of the two repeated tests within patients and the repeatability coefficient were calculated ${ }^{19}$. In estimating the SD of the difference between the two repeated tests, it was assumed that the true mean difference equals zero. The repeatability coefficient has been defined as 1.96 times the SD of differences between repeated measurements, so that plus/minus the repeatability coefficient gives the 2.5 and $97.5 \%$ probability limits, the so-called limits of agreement for the difference between repeated measurements ${ }^{19}$. Further, the coefficient of variation was calculated from the two repeated tests, defined as the SD of the error in a single test and expressed as a percentage of the population mean of the test variable considered. Last, it was tested whether the variability in repeated tests, defined as the absolute value of the difference between the tests, was related to gestational age and FHR.

\section{RESULTS}

Technically acceptable flow velocity waveforms from the middle and distal pulmonary veins were collected in 96 $(86 \%)$ and $81(73 \%)$, respectively, out of 111 women. Recording failures were determined by fetal (breathing) movements, unfavorable fetal position or maternal obesity. There was no relationship between the recording failures and the left or right lung or gestational age, although recording failures seemed to occur slightly more often after 35 weeks of gestation, owing to fetal breathing movements. The median gestational age at delivery was 40 weeks (range 36-42 weeks) and the median birth weight was $3190 \mathrm{~g}$ (range 1930-4760 g).

\section{Waveform characteristics}

Proximal, middle and distal venous pulmonary flow velocity waveforms are demonstrated in Figure 1 . The nature of the middle and distal venous pulmonary branch blood flow velocity waveform exhibited a profile comparable with that of the proximal venous pulmonary branch ${ }^{11}$, indicating a biphasic pattern consisting of a systolic and early diastolic forward flow component and forward flow during late diastole. However, in $3 \%$ of middle venous pulmonary flow velocity waveforms and in $16 \%$ of distal venous pulmonary flow velocity waveforms, no end-diastolic forward flow could be observed. Since the lowest wall filter setting was $70 \mathrm{~Hz}(1.44 \mathrm{~cm} / \mathrm{s})$, flow velocities lower than 1.44 $\mathrm{cm} / \mathrm{s}$ were assumed to be zero.

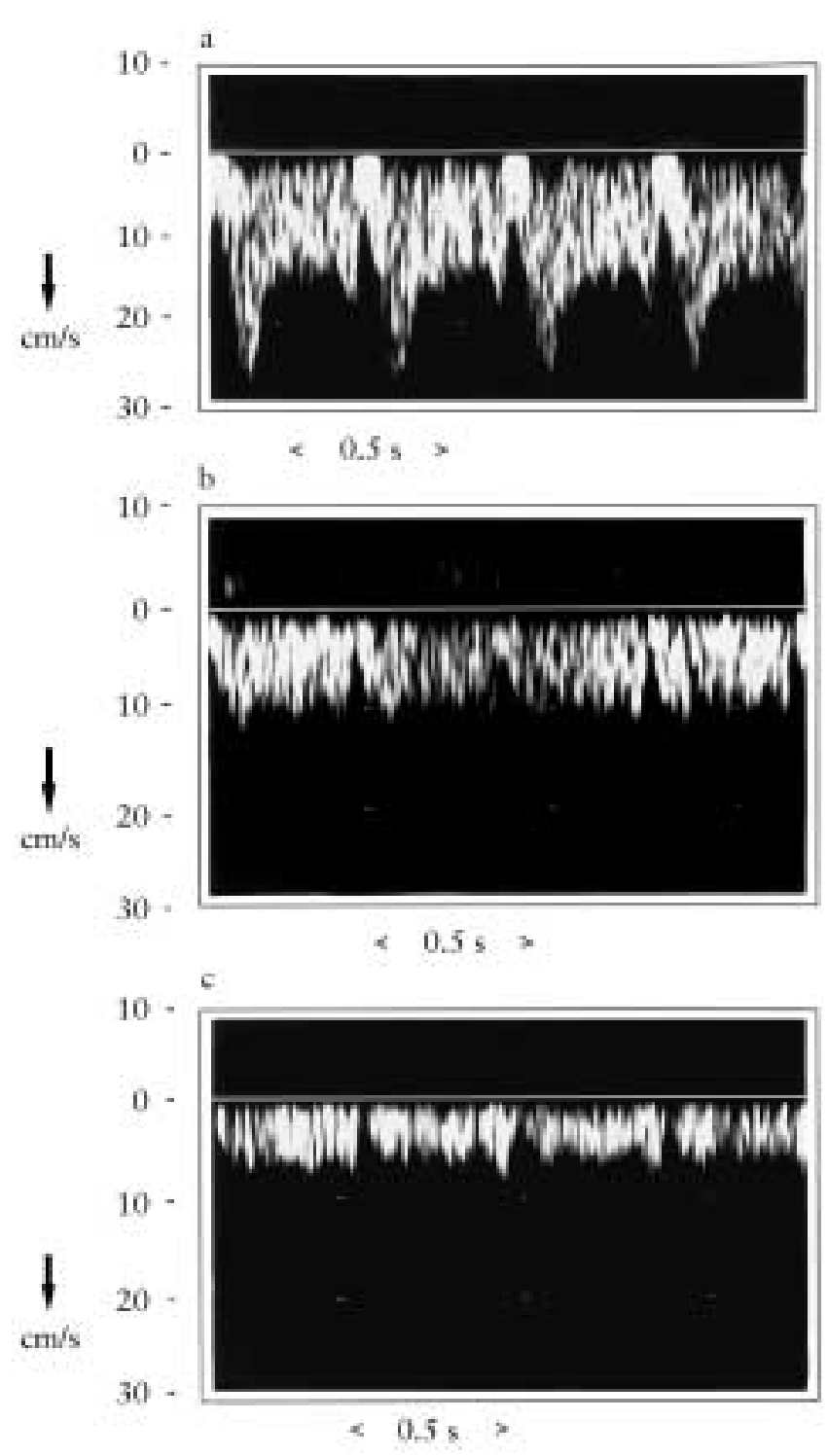

Figure 1 Doppler ultrasound recording of blood flow velocity waveforms from the (a) proximal, (b) middle and (c) distal branches of the pulmonary vein at 25 weeks of gestation

\section{Repeatability}

Outcomes of repeatability are demonstrated in Table 1. The mean differences of the two repeated tests were not statistically significant. The differences of the two repeated measurements were not related to the mean of both tests. All absolute differences between the two repeated tests were not significantly related to fetal heart rate. Since the homogeneity of variances between the two gestational age groups was not significantly different at the $1 \%$ level using the $F$ test, all data of the 25 patients could be combined; a significance level of $1 \%$ was used because of multiple testing.

For the proximal venous pulmonary branch waveform parameters, the coefficient of variation $(\mathrm{CV})$ varied from 6 to $13 \%$ (lowest, peak diastolic velocity; highest, enddiastolic velocity). For the middle venous pulmonary branch waveform parameters, the $\mathrm{CV}$ ranged between 
6 and $23 \%$ (lowest, S/D ratio; highest, end-diastolic velocity) and for the distal venous pulmonary branch waveform, between 5 and 24\% (lowest, S/D ratio; highest, enddiastolic velocity).

Table 1 Outcomes of repeatability of Doppler recordings from the fetal proximal, middle and distal venous pulmonary branches in 25 pregnancies

\begin{tabular}{|c|c|c|c|c|}
\hline & $M D$ & $\begin{array}{c}\text { SD of } \\
\text { differences }\end{array}$ & $\begin{array}{c}\text { Repeatability } \\
\text { coefficient }\end{array}$ & $C V(\%)$ \\
\hline \multicolumn{5}{|c|}{ Proximal venous pulmonary branch } \\
\hline $\operatorname{TAV}(\mathrm{cm} / \mathrm{s})$ & 0.22 & 2.00 & \pm 3.92 & 9 \\
\hline $\operatorname{PSV}(\mathrm{cm} / \mathrm{s})$ & 0.04 & 2.55 & \pm 4.99 & 9 \\
\hline $\operatorname{PDV}(\mathrm{cm} / \mathrm{s})$ & -0.16 & 1.66 & \pm 3.26 & 6 \\
\hline $\operatorname{EDV}(\mathrm{cm} / \mathrm{s})$ & -0.10 & 1.19 & \pm 2.34 & 13 \\
\hline $\mathrm{S} / \mathrm{D}$ ratio & -0.01 & 0.10 & \pm 0.20 & 7 \\
\hline PIV & -0.03 & 0.17 & \pm 0.34 & 12 \\
\hline \multicolumn{5}{|c|}{ Middle venous pulmonary branch } \\
\hline $\operatorname{TAV}(\mathrm{cm} / \mathrm{s})$ & 0.31 & 1.58 & \pm 3.09 & 11 \\
\hline $\operatorname{PSV}(\mathrm{cm} / \mathrm{s})$ & 0.26 & 1.53 & \pm 2.99 & 8 \\
\hline $\operatorname{PDV}(\mathrm{cm} / \mathrm{s})$ & -0.01 & 2.12 & \pm 4.16 & 11 \\
\hline $\operatorname{EDV}(\mathrm{cm} / \mathrm{s})$ & -0.20 & 1.12 & \pm 2.19 & 23 \\
\hline $\mathrm{S} / \mathrm{D}$ ratio & 0.02 & 0.10 & \pm 0.20 & 6 \\
\hline PIV & 0.10 & 0.14 & \pm 0.28 & 10 \\
\hline \multicolumn{5}{|c|}{ Distal venous pulmonary branch } \\
\hline $\operatorname{TAV}(\mathrm{cm} / \mathrm{s})$ & -0.21 & 0.96 & \pm 1.90 & 12 \\
\hline $\operatorname{PSV}(\mathrm{cm} / \mathrm{s})$ & -0.29 & 0.98 & \pm 1.93 & 9 \\
\hline $\operatorname{PDV}(\mathrm{cm} / \mathrm{s})$ & -0.15 & 0.97 & \pm 1.90 & 9 \\
\hline $\operatorname{EDV}(\mathrm{cm} / \mathrm{s})$ & -0.32 & 0.85 & \pm 1.67 & 24 \\
\hline S/D ratio & -0.02 & 0.10 & \pm 0.20 & 5 \\
\hline PIV & 0.01 & 0.17 & \pm 0.34 & 15 \\
\hline
\end{tabular}

$\mathrm{MD}$, mean difference of repeated tests; SD, standard deviation of differences of repeated tests; CV, coefficient of variation; TAV, time-averaged maximum velocity; PSV, peak systolic velocity; PDV, peak diastolic velocity; EDV, end-diastolic velocity; S/D, peak systolic/peak diastolic; PIV, pulsatility index for veins

\section{Venous pulmonary branch velocity parameters relative to gestational age}

The regression equations for Doppler blood flow velocity parameters of middle and distal venous pulmonary branches relative to gestational age are described in Table 2. Statistically significant gestational age-related changes in middle and distal venous pulmonary flow velocity parameters are depicted in Figures 2-5.

For the middle venous pulmonary branches, a statistically significant increase with advancing gestational age was found for all velocity components and the $\mathrm{S} / \mathrm{D}$ ratio, apart from the PIV, which demonstrated a statistically significant reduction with advancing gestation (Figures 2 and 3). A significant increase existed for peak systolic velocity (Figure $2 \mathrm{a}$ ) and TAV (Figure 3a) until 31-32 weeks of gestation and for end-diastolic velocity (Figure 2c) until 33 weeks of gestation. The peak systolic and end-diastolic velocities did not change significantly thereafter until term, whereas the TAV showed a significant reduction from 35 weeks to term. The peak diastolic velocity (Figure 2b) displayed a significant rise from 20 to 31 weeks and a significant decline from 33 weeks until term. The S/D ratio (Figure 3b) increased significantly from 29 weeks until term.

In the distal venous pulmonary branches, the peak systolic velocity (Figure 4a), peak diastolic velocity (Figure $4 \mathrm{~b}$ ), end-diastolic velocity (Figure 4c) and TAV (Figure 5a) demonstrated a statistically significant increase with advancing gestational age. Both TAV and peak diastolic velocity increased significantly until 31 weeks, followed by no significant change until term. The PIV (Figure 5b) displayed a significant decrease, whereas the $S / D$ ratio remained unchanged throughout the second half of pregnancy.

Table 2 Regression equations for various venous pulmonary branch Doppler measurements and their ratios with gestational age (GA)

\begin{tabular}{|c|c|c|c|c|c|c|c|c|}
\hline & \multirow[b]{2}{*}{ Transformation } & \multirow[b]{2}{*}{ Constant } & \multirow[b]{2}{*}{$A \times G A$} & \multirow[b]{2}{*}{$B \times G A^{2}$} & \multicolumn{2}{|c|}{$S D$} & \multirow[b]{2}{*}{$R$} & \multirow[b]{2}{*}{$p$} \\
\hline & & & & & Constant & $C \times G A$ & & \\
\hline \multicolumn{9}{|c|}{ Proximal branch ${ }^{11}$} \\
\hline \multicolumn{9}{|c|}{ Middle branch } \\
\hline $\operatorname{TAV}(\mathrm{cm} / \mathrm{s})$ & - & -17.54 & 1.82 & -0.028 & 1.96 & - & 0.50 & $<0.0001$ \\
\hline $\operatorname{PSV}(\mathrm{cm} / \mathrm{s})$ & - & -14.10 & 1.76 & -0.026 & 2.44 & - & 0.46 & $<0.0001$ \\
\hline $\operatorname{PDV}(\mathrm{cm} / \mathrm{s})$ & - & -23.68 & 2.52 & -0.040 & 2.62 & - & 0.47 & $<0.0001$ \\
\hline S/D ratio & $\log (S / D)$ & 0.43 & -0.037 & $6.9 \times 10^{4}$ & 0.09 & - & 0.34 & 0.003 \\
\hline PIV & $\log (\mathrm{PI})$ & 0.25 & -0.014 & - & 0.23 & - & 0.32 & 0.001 \\
\hline \multicolumn{9}{|c|}{ Distal branch } \\
\hline $\operatorname{TAV}(\mathrm{cm} / \mathrm{s})$ & - & -4.74 & 0.63 & -0.0095 & 1.14 & - & 0.35 & 0.005 \\
\hline PSV $(\mathrm{cm} / \mathrm{s})$ & - & 4.03 & 0.096 & - & 1.35 & - & 0.35 & 0.001 \\
\hline $\operatorname{PDV}(\mathrm{cm} / \mathrm{s})$ & - & -6.03 & 0.83 & -0.013 & 1.48 & - & 0.33 & 0.01 \\
\hline $\operatorname{EDV}(\mathrm{cm} / \mathrm{s})$ & $\log (\mathrm{EDV})$ & 0.53 & 0.016 & - & 0.28 & - & 0.30 & 0.01 \\
\hline PIV & $\log (\mathrm{PI})$ & 0.24 & -0.015 & - & 0.32 & - & 0.24 & 0.03 \\
\hline
\end{tabular}

A, coefficient for linear components; B, coefficient for quadratic component; C, coefficient for linear component if the absolute residuals depended on GA; SD, residual standard deviation (as a linear function of GA if necessary); R, multiple correlation coefficient; $p$, significance of regression; TAV, time-averaged maximum velocity; PSV, peak systolic velocity; PDV, peak diastolic velocity; S/D, peak systolic/peak diastolic; PIV, pulsatility index for veins; *, when the regression was not significant, the mean and SD are presented; NS, not significant 

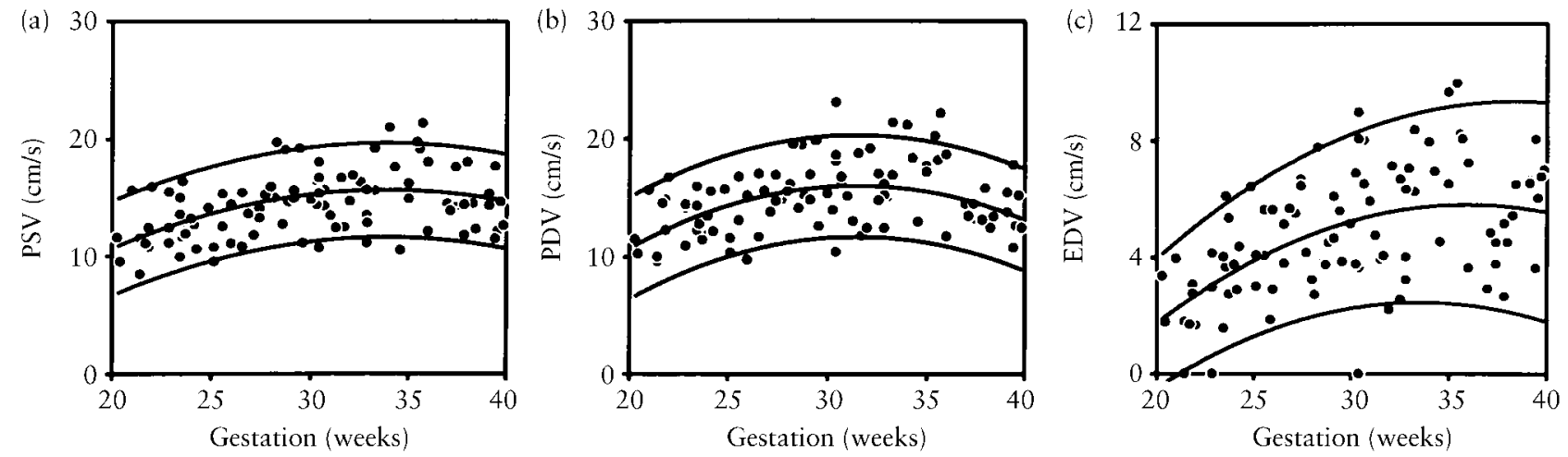

Figure 2 Individual values and reference ranges (mean, 5th and 95th centiles) for (a) peak systolic velocity (PSV), (b) peak diastolic velocity (PDV) and (c) end-diastolic velocity (EDV) of the middle venous pulmonary branch relative to gestational age
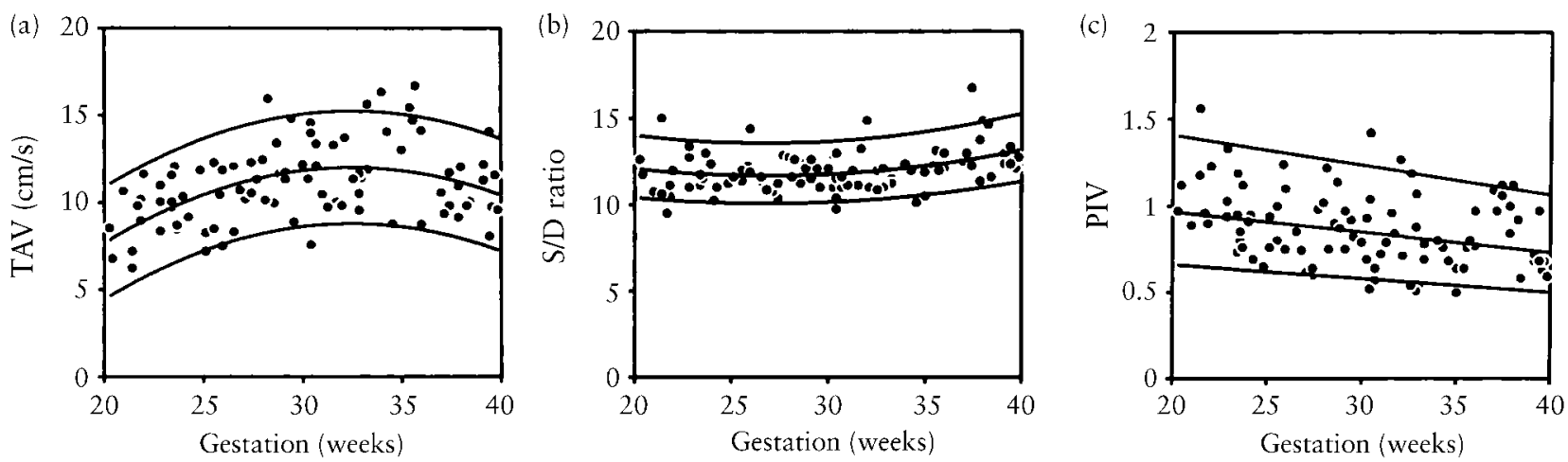

Figure 3 Individual values and reference ranges (mean, 5th and 95th centiles) for (a) time-averaged maximum velocity (TAV), (b) peak systolic/peak diastolic (S/D) ratio and (c) pulsatility index (PIV) of the middle venous pulmonary branch relative to gestational age
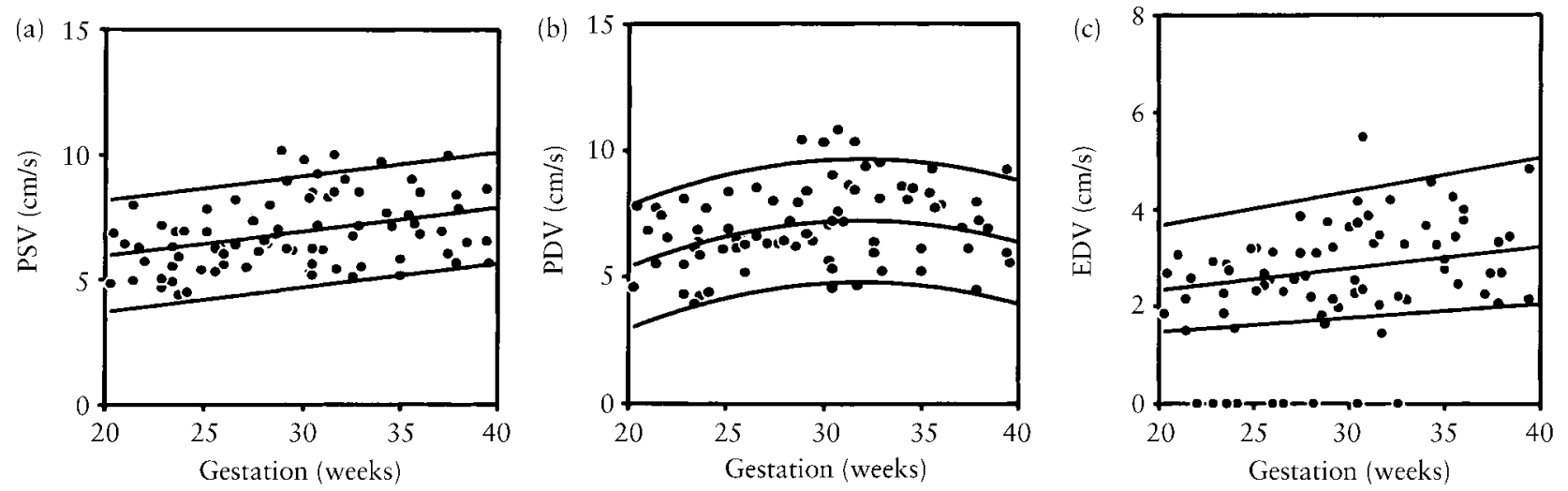

Figure 4 Individual values and reference ranges (mean, 5th and 95th centiles) for (a) peak systolic velocity (PSV), (b) peak diastolic velocity (PDV) and (c) end-diastolic velocity (EDV) of the distal venous pulmonary branch relative to gestational age

The observed relationship between the venous flow velocity waveform parameters, PIV calculations of both middle and distal pulmonary branches and gestational age was independent of FHR.

Inter-pulmonary (proximal, middle, distal) differences in venous blood flow velocity parameters

All mean differences in the Doppler flow velocity parameters between proximal and middle, between proximal and distal, and between middle and distal venous pulmo- nary branches were statistically significant, apart from the mean difference in PIV between middle and distal venous pulmonary flow velocity waveforms, which was not significant (Table 3).

No significant gestational age-related change existed for mean differences in:

(1) Peak systolic velocity, peak diastolic velocity, PIV and S/D ratio between middle and distal venous pulmonary branches;

(2) End-diastolic velocity and PIV between proximal and middle venous branches; 

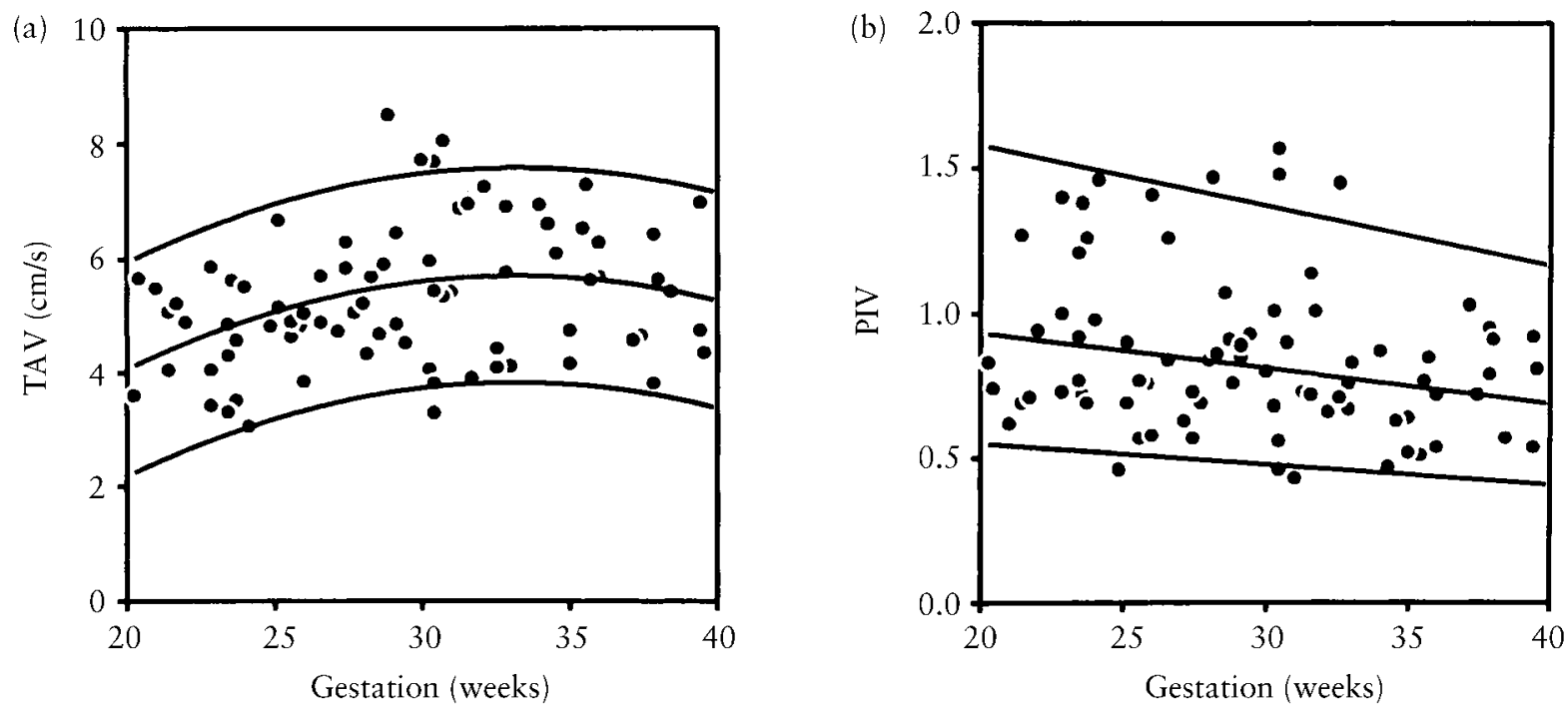

Figure 5 Individual values and reference ranges (mean, 5th and 95th centiles) for (a) time-averaged maximum velocity (TAV) and (b) pulsatility index (PIV) of the distal venous pulmonary branch relative to gestational age

Table 3 Differences in Doppler flow velocity parameters from proximal, middle and distal venous pulmonary branches in the second half of gestation

\begin{tabular}{lccc}
\hline & $\begin{array}{c}\text { Number } \\
\text { of pairs }\end{array}$ & MD* & Relationship between \\
MD and GA
\end{tabular}

$\mathrm{MD}$, mean difference; SD, standard deviation; GA, gestational age; *, all MD were statistically significant $(p<0.05)$ except the MD in the PIV $(* *)$ between middle and distal venous branches; ${ }^{\dagger}$, negative correlation with gestational age; S/D, peak systolic/peak diastolic; NS, not significant

(3) PIV between proximal and distal venous branches (Table 3).

Mean differences in S/D ratio between proximal and middle, and between proximal and distal branches of the pulmonary vein revealed a significant decrease, whereas all other remaining mean differences displayed a significant rise with advancing gestational age (Table 3 ).

\section{DISCUSSION}

The present study describes the characteristics and repeatability of Doppler flow velocity waveforms obtained from different locations of the human fetal pulmonary venous circulation during the second half of normal pregnancy. The cross-sectional study design adopted in the present study puts some limitation on the interpretation of the 
collected data. Venous pulmonary blood flow velocity waveforms obtained from one of the pulmonary veins just proximal to the entrance of the left atrium of the human fetus display a biphasic forward flow profile with a systolic and diastolic component reflecting dynamic changes in left atrial pressure as a result of atrial and ventricular contraction and relaxation ${ }^{5,9,11}$. These flow velocity waveform characteristics continue to be present in both middle and distal venous pulmonary branches, although with lower peak velocities. Only in a few cases could no end-diastolic flow be detected in the middle and distal venous pulmonary branches during atrial contraction. A late diastolic reverse flow component at pulmonary venous orifice level has been reported in $38 \%{ }^{12}$ and $18 \%$ of cases ${ }^{13}$. This was never seen in the present study, whether at proximal, middle or distal venous level. Both the sampling site, i.e. immediately proximal to the entrance into the left atrium, and FHR may play a role, since both measurements at the venous orifice level and at lower heart rates as seen in infants ${ }^{6}$ were associated with well-defined late diastolic reverse flow. Alternatively, the wall filter setting of $70 \mathrm{~Hz}$ $(1.44 \mathrm{~cm} / \mathrm{s})$ may have hidden minimal end-diastolic (reverse) flow in some instances. To our knowledge, no other reports are available on Doppler velocimetry from more peripheral venous pulmonary branches.

The coefficients of variation for all velocity parameters, S/D ratios and PIV were below $15 \%$ for proximal, middle and distal branches, apart from the end-diastolic velocity of middle and distal venous pulmonary flow velocity waveforms $(23 \%$ and $24 \%$, respectively). Overall, the parameters from the proximal branch flow velocity waveform showed the lowest coefficients of variation. However, to judge repeatability for clinical practice, measurement variability expressed as plus/minus the repeatability coefficient should be taken into account; $95 \%$ of the differences between two repeated tests in the same arbitrary patient will lie within this interval. This variability may be due to within-patient as well as intra-observer variation. There is only one other report ${ }^{13}$ that has studied repeatability, although only for waveforms obtained from the proximal venous pulmonary branches and not from more distal branches. Unfortunately, it is not possible to compare our repeatability data with the outcomes from that particular study, since it did not report from which velocity parameters the recounted mean differences were calculated $^{13}$.

In a previous study ${ }^{11}$, a gestational age-dependent rise in flow velocities (TAV, peak systolic, peak diastolic and enddiastolic velocities) was established from the proximal venous pulmonary branches. It was speculated that an increase in volume flow and pressure gradient between the pulmonary venous system and the left atrium may play a role in this ${ }^{11}$. Rasanen and colleagues ${ }^{20}$ demonstrated in the human fetus an increase from 13 to $25 \%$ in the proportion of pulmonary flow when related to combined cardiac output at 20-30 weeks of gestation. This proportion remained unchanged during the remainder of pregnancy. This is consistent with the present observation that, for the middle and distal venous pulmonary branches, a gestational age- dependent rise in flow velocities was obtained for all waveform velocity parameters, mainly evident in the second trimester and first part of the third trimester.

The pulsatile pattern of flow velocity waveforms in the pulmonary venous system is the result of changes in pressure difference between the pulmonary venous system and the left atrium $^{21}$. Based on the linear decrease in PIV for both the middle and distal venous pulmonary branches with gestation, an increase in pulmonary venous pressure gradient is suggested. This may be the result of a decline in proportion of volume flow through the foramen ovale ${ }^{20}$ and/or a decrease in cardiac afterload, due to a reduction in placental resistance ${ }^{1,21}$. The decrease in cardiac afterload causes a decrease in end-diastolic ventricular pressure and therefore an increase in blood flow velocity during atrial contraction. This is demonstrated by the significant increase in end-diastolic velocity in the proximal ${ }^{11}$, middle and distal venous pulmonary branches. Unfortunately, we cannot compare our outcomes on middle and/or distal human fetal venous pulmonary hemodynamics with other studies, since these, to our knowledge, are not available.

When the inter-relationship between proximal, middle and distal arterial pulmonary flow velocity waveforms was considered, all velocity parameters demonstrated a significant change from proximal to distal level. The mean peak systolic and peak diastolic velocities dropped 1.8- and 1.6fold from proximal to middle and 3.7- and 3.4-fold from proximal to distal, whereas the mean end-diastolic velocity dropped 1.9-fold and 3.8-fold, respectively. Changes in vessel diameter, increase in vessel branching and increase in distance between the heart and distal venous pulmonary vessels may account for these observations ${ }^{22}$. Although the PIV remained unchanged as pulsed Doppler interrogation proceeded from middle to distal, it changed significantly from proximal to middle and from proximal to distal. The mean differences of PIV between the three locations were not related to gestational age. This suggests that the pulsatility of the venous pulmonary system is also influenced by the distance between the heart and the site of Doppler interrogation and/or vessel diameter.

It can be concluded that color-coded Doppler systems allow reliable Doppler velocity waveform recordings to be obtained in the more distal parts of the human fetal pulmonary venous system during the second half of pregnancy. Pulmonary venous flow velocity waveforms are not essentially different at the proximal, middle or distal levels. Acceptable repeatability of flow velocity waveforms with coefficients of variation below $15 \%$ was established for nearly all velocity parameters and their ratios. A gestational age-dependent change was found for all flow velocity waveform parameters including PIV at both middle and distal level. It is speculated that increases in volume flow and venous pulmonary pressure gradient play a role in these findings. Nearly all waveform parameters alter significantly as pulsed Doppler interrogation proceeds from proximal to more distal venous lung regions. This may be due to changes in vessel diameter and distance between the heart and more distal venous pulmonary vessels. 


\section{REFERENCES}

1. Rudolph AM, Heymann MA. Circulatory changes during growth in the fetal lamb. Circ Res 1970;26:289-99

2. Lewis AB, Heymann MA, Rudolph AM. Gestational changes in pulmonary vascular responses in fetal lambs in utero. Circ Res 1976;39:536-41

3. Morin FC III, Egan EA. Pulmonary hemodynamics in fetal lambs during development at normal and increased oxygen tension. J Appl Physiol 1992;73:213-18

4. Ramjagopalan B, Friend JA, Stallard T, Lee G de J. Blood flow in pulmonary veins: 1 . Studies in $\operatorname{dog}$ and man. Cardiovasc Res 1979;13:667-76

5. Keren G, Sherez J, Megidish R, Levitt B, Laniado S. Pulmonary venous flow pattern - its relationship to cardiac dynamics. A pulsed Doppler echocardiography study. Circulation 1985;71:1105-12

6. Smallhorn JF, Freedom RM, Olley PM. Pulsed Doppler echocardiography assessment of extraparenchymal pulmonary vein flow. J Am Coll Cardiol 1987;9:573-9

7. Angelini A, Allan L, Anderson RH, Crawford DC, Chita SK, Yen Ho S. Measurements of the dimensions of the aortic and pulmonary pathways in the human fetus: a correlative echocardiographic and morphometric study. Br Heart J 1988;60: 211-16

8. DeVore GR. The use of color Doppler imaging to examine the fetal heart. Normal and pathologic anatomy. In Jaffe R, Warsof SI, eds. Color Doppler Imaging in Obstetrics and Gynecology. New York: McGraw-Hill, 1992:126-9

9. Emerson DS, Cartier MS. The fetal pulmonary circulation. In Copel JA, Reed KL, eds. Doppler Ultrasound in Obstetrics and Gynecology. New York: Raven Press, 1995:307-23

10. Anteby EY, Shimonovitz S, Yagel S. Fetal echocardiography: the identification of two of the pulmonary veins from the four-chamber view during the second trimester of pregnancy. Ultrasound Obstet Gynecol 1994;4:208-10
11. Laudy JAM, Huisman TWA, de Ridder MAJ, Wladimiroff JW. Normal fetal pulmonary venous blood flow velocity. Ultrasound Obstet Gynecol 1995;6:277-81

12. Better DJ, Kaufman S, Allan L. The normal pattern of pulmonary venous flow on pulsed Doppler examination of the human fetus. J Am Soc Echocardiogr 1996;9:281-5

13. Paladini D, Palmieri S, Celentano E, Guida F, Salviati M, Morra T, Martinelli P. Pulmonary venous blood flow in the human fetus. Ultrasound Obstet Gynecol 1997;10:27-31

14. Kloosterman G. On intrauterine growth. Int Gynecol Obstet 1970;8:895-912

15. Maršál K, Lindblad A, Lingman G. Blood flow in the fetal descending aorta; intrinsic factors affecting fetal blood flow, i.e. fetal breathing movements and cardiac arrhythmia. Ultrasound Med Biol 1984;10:339-48

16. Gosling RG, King DH. Ultrasound angiology. In Marcus AW, Adamson J, eds. Arteries and Veins. Edinburgh: ChurchillLivingstone, 1975:61-98

17. Royston P. Constructing time-specific reference ranges. Stat Med 1991;10:675-90

18. Altman D. Construction of age-related reference centiles using absolute residuals. Stat Med 1993;12:917-24

19. Bland JM, Altman DG. Statistical methods for assessing agreement between two methods of clinical measurement. Lancet 1986;2:307-10

20. Rasanen J, Wood DC, Weiner S, Ludomirski A, Hutha JC. Role of the pulmonary circulation in the distribution of human fetal cardiac output during the second half of pregnancy. Circulation 1996;94:1068-73

21. Hecher K, Campbell S, Snijders R, Nicolaides K. Reference ranges for fetal venous and atrioventricular blood flow parameters. Ultrasound Obstet Gynecol 1994;4:381-90

22. Hislop A, Reid L. Fetal and childhood development of the intrapulmonary veins in man - branching pattern and structure. Thorax 1973;28:313-19 